\title{
Persistent Oxidative Stress after Myocardial Infarction Treated by Percutaneous Coronary Intervention
}

\author{
Vjeran Nikolic-Heitzler, Filip Rabuzin, ${ }^{1}$ Franz Tatzber, ${ }^{2}$ Nada VRkic, \\ Nikola Bulj, Suzana Borovic, ${ }^{1}$ Willibald Wonisch, ${ }^{3}$ Branka Mazul Sunko \\ and Neven Zarkovic ${ }^{1}$ \\ University Clinical Hospital "Sestre milosrdnice", Zagreb, Croatia, \\ 1 "Rudjer Boskovic" Institute, Division of Molecular Medicine, Zagreb, Croatia, \\ ${ }^{2}$ Department of Nuclear Medicine, University of Vienna, Austria, and \\ ${ }^{3}$ Clinical Institute of Medical and Chemical Laboratory Diagnostics, Medical \\ University, Graz, Austria
}

Nikolic-Heitzler, V., Rabuzin, F., Tatzber, F., Vrkic, N., Bulj, N., Borovic, S., Wonisch, W., Sunko, B.M. and Zarkovic, N. Persistent Oxidative Stress after Myocardial Infarction Treated by Percutaneous Coronary Intervention. Tohoku J. Exp. Med., 2006, 210 (3), 247-255 — Acute myocardial infarction causing cardiac ischemia is responsible for the majority of cardiac related deaths. Medical interventions that ensure rapid reperfusion, such as percutaneous coronary intervention, are aimed to allow myocardial re-oxygenation. However, this generates reactive oxygen species, resembling ischemiareperfusion type of injury based on oxidative stress. In the present study we monitored dynamic changes of total serum peroxides, total antioxidant capacity and soluble intercellular adhesion molecule-1 as well as the titer of antibodies against oxidized low-density lipoproteins in the blood during the convalescence period of 32 patients with acute myocardial infarction treated by percutaneous coronary intervention. Samples were taken at admittance and at two hours, four hours, three days and seven days following percutaneous coronary intervention. Total antioxidant capacity dropped to $82 \%(p<0.05)$. The titer of antibodies against oxidized low-density lipoproteins transiently decreased within the first three days, and increased afterwards. The values of serum peroxides and soluble intercellular adhesion molecule-1 increased continuously in respect to the initial levels reaching the maximum at the time of release from hospital. These findings indicate a persistent oxidative stress that might be associated with intravascular inflammation in patients during convalescence and release from hospital. —— myocardial infarction; percutaneous coronary intervention; inflammation; soluble intercellular adhesion molecule-1; lipid peroxidation

(C) 2006 Tohoku University Medical Press

While oxidative stress has been shown to be related with the age and living habits (Ozbay and Dulker 2002), it plays an important role in the development of vascular diseases that affect vital organs, in particular brain and heart (Zarkovic 2003; Zarkovic et al. 2004; Madamanchi et al.

Received June 7, 2006; revision accepted for publication September 22, 2006.

Correspondence: Neven Zarkovic, M.D., Ph.D., Senior Scientist, Rudjer Boskovic Institute, Bijenicka 54, HR-1000 Zagreb, Croatia.

e-mail: zarkovic@irb.hr 
2005; Bir et al. 2006).

Acute myocardial infarction (AMI) is the most critical event in cardiovascular disorders and arises as a consequence of myocardial ischemia due to coronary artery occlusion. Reperfusion therapy is a contemporary, effective treatment of patients with acute ST-segment elevation myocardial infarction (STEMI). Numerous studies have shown a clear superiority of primary percutaneous coronary intervention (PCI) over pharmacological thrombolysis for the treatment of STEMI with higher initial reperfusion rates and better longterm clinical outcomes. The performance of primary PCI is however scarcely performed, hence, only $25 \%$ of AMI cases in the USA and $10 \%$ in Europe are treated by PCI.

Reactive oxygen species (ROS) play an essential role in the pathophysiological myocardial processes during ischemia and reperfusion. The action of ROS and the ensuing lipid peroxidation exhaust the organism's antioxidant capacity (Frei 1994). During early reperfusion oxidative stress might also lead to myocardial stunning and arrhythmia (Ferrari et al. 1993; Akar et al. 2005). The reaction of ROS with polyunsaturated fatty acids (PUFA) of low-density lipoproteins (LDL) leads to the formation of oxidized LDL (oLDL). Reactive aldehydes, which represent the end products of the lipid peroxidation cascade, such as malondialdehyde and 4-hydroxynonenal, may damage the endothelial layer in the vascular system (Steinbrecher 1987; Zarkovic 2003). These lipid peroxidation products covalently bind to apolipoprotein B (apoB) of LDL forming immunogenic epitopes that bind to the scavenger receptors on macrophages (Jürgens et al. 1987). Therefore, the titer of antibodies against oLDL (oLAb) directed against oxidatively modified epitopes on apoB of oLDL has also been shown to be a reliable predictor for the progression of atherosclerosis within the carotid arteries (Vaarala 2000).

On the other hand, atherosclerosis and consequential cardiovascular diseases are also considered as chronic inflammatory process, in which C-reactive protein seems to be associated with the severity of atherosclerosis, while soluble intercel- lular adhesion molecule-1 (sICAM-1) levels are associated with carotid plaques (van der Meer et al. 2002).

The purpose of this study was to investigate the appearance of oxidative stress and the inflammation of coronary arteries during the convalescence period in patients suffering from AMI who had undergone PCI. We therefore monitored the dynamic changes of total serum peroxides, total antioxidant capacity and SICAM-1 as well as the titer of oLAb during the course of convalescence of 32 patients with AMI treated by PCI.

\section{Patients and Methods}

\section{Patients}

The study was performed according to the Helsinki declaration. The Ethics Committee of the University Hospital "Sestre milosrdnice" approved the protocol and written, informed consent was obtained from all patients.

After established diagnosis of AMI in the Emergency Unit of the University Hospital "Sestre milosrdnice" in Zagreb, all patients (Table 1) were immediately transferred to the catheter laboratory. Patients were admitted within 6 hours following the development of symptoms. Criteria for diagnosis and urgent PCI were established following recent ESC guidelines for AMI with STEMI diagnosis and treatment. Patients were treated with oxygen, nitro-glycerine, aspirin $(330 \mathrm{mg})$, a loading dose of ticlopidine $(500 \mu \mathrm{g})$ and $5,000 \mathrm{IU}$ bolus of heparin followed by $18 \mathrm{IU} / \mathrm{kg} / \mathrm{h}$ prior to the intervention. It is assumed that such a routine medicamentous treatment should not have a major impact on the parameters analysed in this study during a week of convalescence. Patients were admitted to the Coronary Care Unit following successful primary PCI.

As AMI differs from stable angina pectoris in many pathological aspects, it is commonly preferred to consider AMI patients at admission as their own effective control for the dynamic change of parameters followed within a particular period of time, instead of comparing them with patients suffering from angina pectoris. Therefore, the values obtained at the admission were considered as relevant control values for parameters analyzed during the period of post-AMI and PCI convalescence.

\section{Sample preparation}

Blood was initially taken from the cubital vein just prior to primary PCI after medicamentous treatment as described before. The other samples of blood taken from 
TABLE 1. Description of patients.

\begin{tabular}{lc}
\hline & $\begin{array}{c}\text { Patients } \\
(n=32)\end{array}$ \\
\hline Age (yr) & $60( \pm 11)$ \\
Male/Female & $24 / 8$ \\
Smokers/Non smokers & $23 / 9$ \\
Cigarettes consumed per day & $16( \pm 3)$ \\
MI site (ant/inf/lat) & $11 / 19 / 2$ \\
& \\
LDL (mM) & $3.35( \pm 0.28)$ \\
HDL (mM) & $0.93( \pm 0.07)$ \\
Triglycerides (mM) & $1.93( \pm 0.81)$ \\
& \\
Fibrinogen $(\mathrm{g} / \mathrm{l})$ & $2.03( \pm 0.4)$ \\
& \\
Ejection fraction $(\%)$ & $51.76( \pm 0.22)$ \\
(Simpson) & \\
CK (initial/max) $\left(\mathrm{U} / 1,37^{\circ} \mathrm{C}\right)$ & $117.97 / 2,146.21$ \\
AST (initial/max) $\left(\mathrm{U} / 1,37^{\circ} \mathrm{C}\right)$ & $27.82 / 278.47$ \\
ALT (initial/max) $\left(\mathrm{U} / 1,37^{\circ} \mathrm{C}\right)$ & $29.18 / 141.50$ \\
LDH (initial/max) $\left(\mathrm{U} / 1,37^{\circ} \mathrm{C}\right)$ & $319.38 / 932.44$ \\
\hline
\end{tabular}

CK, AST, ALT, LDH, LDL, HDL, triglycerides, fibrinogen and daily consumption of cigarettes. Values are mean values from all patients \pm S.E.M.

MI, myocardial infarction; ant/inf/lat, anterior/inferior/lateral; CK, creatine kinase; AST, aspartate aminotransferase; ALT, alanine aminotransferase; LDH, lactate dehydrogenase; LDL, low-density lipoprotein; HDL, high-density lipoprotein; $n$, number of patients.

the cubital vein were obtained at two hours, four hours, three days and seven days following PCI. On the third and the seventh day after PCI, blood samples were prepared at the same time, (between 6 and 7 a.m.). Blood taken from patients was collected in a centrifuge tube and the sample was left to clot for $30 \mathrm{~min}$ and was then spun down at $1,500 \mathrm{rpm}$ for $15 \mathrm{~min}$. Aliquots were stored at $-80^{\circ} \mathrm{C}$ until analysis.

\section{Total antioxidant capacity}

The antioxidant capacity of sera was determined by a commercially available method $\left(\right.$ TAC $^{\circledR}-$ LDN, Nordhorn, Germany) as previously described (Resch et al. 2006). The assay is based on the consumption of antioxidants present in the sample after the addition of hydrogen peroxide. Serial dilutions of Trolox (6hydroxy-2,3,8-tetramethylchroman-2-carboxylic acid) were used for standardisation. Results were expressed as mmol $1^{-1}$ Trolox equivalents.

\section{Total serum peroxides}

Serum peroxide concentrations were determined by a rapid enzymatic, in vitro diagnostic assay (TOC - LDN) as previously described (Tatzber et al. 2003). The assay is based on a peroxide/peroxidase reaction using tetramethyl-benzidine (TMB) as the chromogenic substrate. Peroxide levels are expressed as " $\mu \mathrm{M} \mathrm{H}_{2} \mathrm{O}_{2}$ equivalents", because of different contributions of various peroxides to the reaction. The inter and intra assay variance was $4.5 \%$.

\section{Titer of antibodies to oxidized LDL (oLAb)}

The titer of antibodies against oLDL was measured in sera with a commercial enzymatic immunoassay (Biomedica, Vienna, Austria) as previously described (Tatzber and Esterbauer 1995). The assay is based on the binding reaction of $1: 50$ diluted samples to the previously oxidized LDL by cupric ions bound to the microtiter wells. Detection was performed by the binding of a secondary, peroxidase coupled anti-IgG antibody, which permitted the colorimetric detection of this enzyme with TMB as the chromogenic substrate. Results were expressed as $\mathrm{mU} / \mathrm{ml}$. The variation coefficient was $6.7 \%$ within a run and $10.9 \%$ from run to run.

\section{sICAM-1}

Reagents were prepared according to the manufacturer's instructions (R\&D systems, Minneapolis, MN, USA). One hundred microlitres of the reagent mixture were added to the micro wells of 96-well plates followed by $100 \mu \mathrm{l}$ of standards or sera samples. The plates were then incubated for $90 \mathrm{~min}$. After the incubation, the plate was washed six times with $300 \mu 1$ of provided wash buffer. One hundred microlitres of the dye substrate (TMB) was added to the wells immediately after the washing step and the plate was incubated for $30 \mathrm{~min}$. The optical density was determined by reading the absorption at 450 $\mathrm{nm}$.

\section{Ejection fraction coefficient}

The extent of the infarction was estimated using a bedside two dimensional Doppler echocardiogram (Acuson SEQUOIA, Erlangen, Germany) within the first 
$24 \mathrm{hrs}$ following admission. The ejection fraction was measured using Simpson's method. Regional wall motion abnormality was estimated by dividing the ventricle into 16 segments in accordance with the recommendations of the American Society of Echocardiography (Crequeira et al. 2002).

\section{Statistical analysis}

SPSS 11.0 program for Windows was used. The statistical significance of the levels of total peroxides, the antioxidant capacity, sICAM-1 and oLAb was calculated using the Chi-square test. Significant differences between these assays at various time points were determined by the Mann Whitney's U-test. Values of $p<0.05$ were considered to be significant. Similarity of dynamic changes between SICAM-1 and serum peroxides was analysed using the Pearson correlation coefficient.

\section{Results}

\section{Total antioxidant capacity}

The total antioxidant capacities of sera of the patients were within the range of normal values most of the time during convalescence (Fig. 1A). Two hours following PCI, the antioxidant capacity decreased to $87 \%$ of the initial value $(p<0.05)$, increasing after three days to the levels measured at the time prior to PCI $(102 \%)$. The values determined seven days following PCI were again
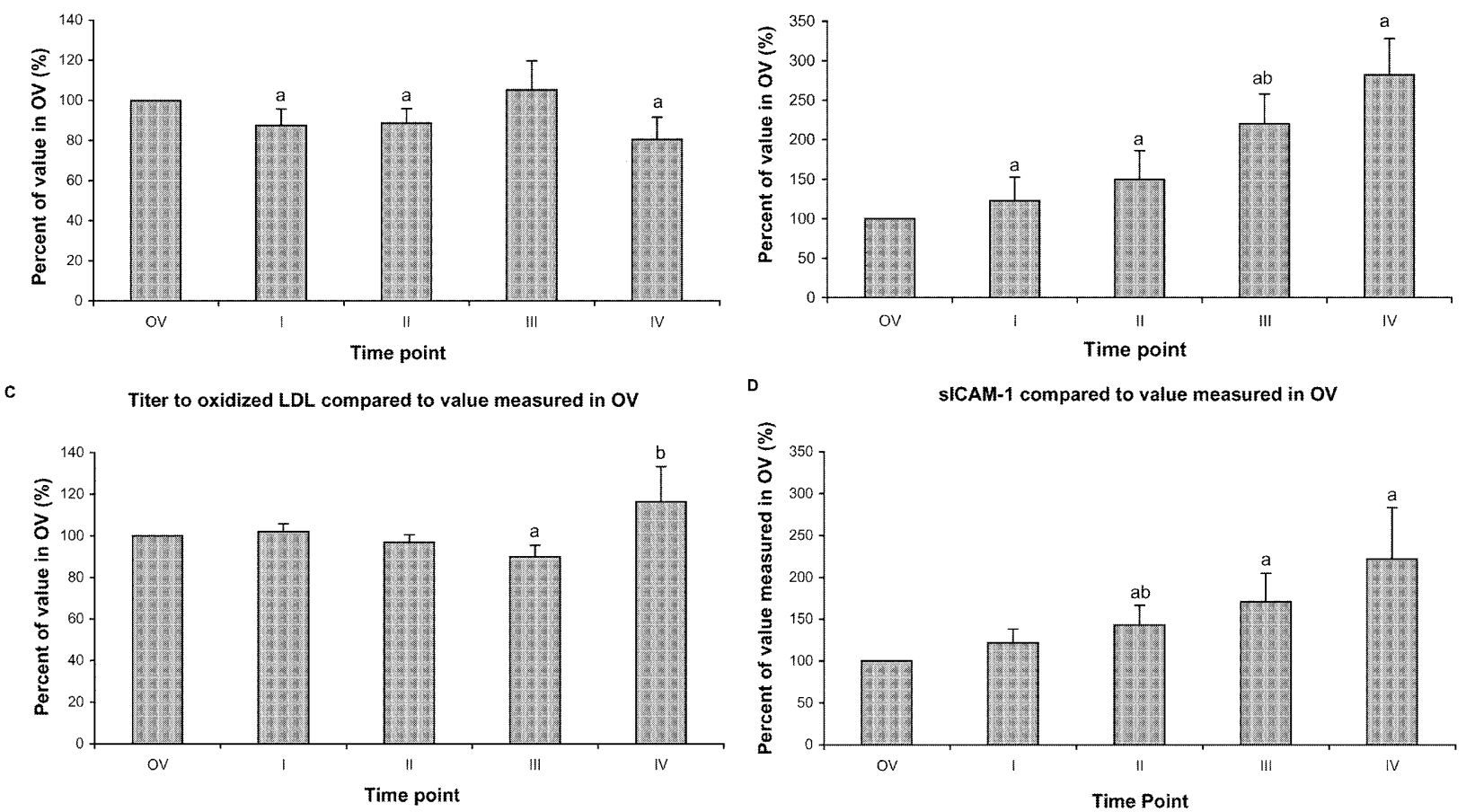

Fig. 1. Evolution of total antioxidant capacity (A), total serum peroxides (B), titer of antibodies to oxidized LDL (C) and sICAM-1 (D) levels.

Significant differences observed between the particular time points and the initial values at admission $(\mathrm{OV})$ are denoted by "a" on the graphs, while significant differences between any time point and the preceding time point are denoted by "b". Results are shown as a percentage of the respective initial values measured at admission, i.e., before the PCI. Blood samples were taken from patients at admission, i.e., prior to PCI (OV), two hours (I), four hours (II), three days (III) and seven days (IV) after PCI.

While serum peroxide and sICAM-1 levels continuously raised, the oLAb results have shown a moderate and transient decrease followed by the raise of the antibody titer typical for successful convalescence after AMI. Total antioxidant capacity of serum has also shown a transient decrease after PCI, which occurred afterwards also on the seventh day. 
significantly lower $(p<0.01)$ than those at admission ( $82 \%$ of initial measurement), still remaining within the range of normal values.

\section{Total peroxides}

Total peroxides (Fig. 1B) were above normal values from the time of admittance. The values of serum peroxides were increasing constantly during the observation period, reaching a maximum at the time of release from hospital (time point IV), which was nearly 3 times increased if compared to the initial peroxide levels $(p<0.01)$.

\section{Titer of antibodies to oxidized LDL}

The titer of antibodies against oLDL (Fig. 1C) was within the range of normal values during the observation period. A tendency of slight and transient decrease (to $89 \%$ of the initial values) was noticed on the third day $(p<0.05)$. On the seventh day, the oLAb values increased slightly in comparison to the values determined initially $(116 \%)$. The increase was found to be significant $(p<0.05)$ between the third and the seventh day of convalescence.

\section{SICAM-1}

Soluble intercellular adhesion molecule-1 levels (Fig. 1D), similar to the serum peroxides, increased from 2 hours to seven days following PCI. The increase was continuous and reached a maximum (220\% of initial values) on the seventh day. The values observed two hours after PCI as well as those obtained from the blood samples taken on the third and on the seventh day were also significantly different from those measured at admittance $(p<0.05)$.

Similarity of raise of SICAM-1 and serum peroxides was also verified by the Pearson correlation coefficient factor of $0.92(p=0.001)$ determined between the evolution pattern of total serum peroxides and sICAM-1 (Fig. 2).

Evaluation of the patterns of dynamic changes of analyzed parameters is presented in Fig. 3. The predominant trend for sICAM-1 and serum peroxides was a raise (for both, $p<0.05$ ). Opposite to that, predominant trend for total

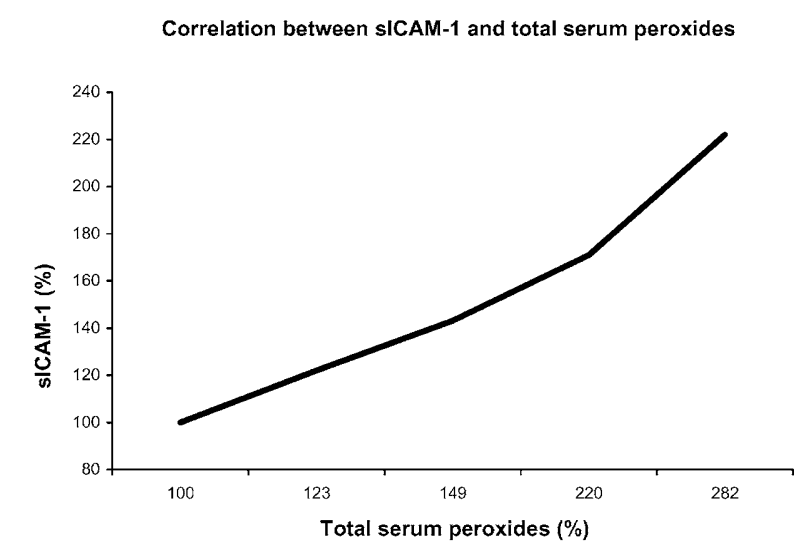

Fig. 2. Correlation between the evolution of sICAM-1 and total serum peroxides levels. The evolution of the two markers in the patient population showed an almost interdependent evolution throughout the observation time giving a Pearson correlation coefficient of 0.92 $(p=0.001)$.

capacity of antioxidants was decrease $(p<0.05)$, while the antibody titer to oLDL predominantly followed a trend of transient fall.

\section{Discussion}

While myocardial damage is known as pathological condition associated with oxidative stress, the results obtained in this study indicate persistent oxidative stress also during convalescence in patients with AMI treated by PCI. Namely, a sustained and significant increase of total peroxides led to a maximum at the time of release from hospital (day seven). The increase of serum peroxides was associated with a decrease in total antioxidant capacity.

Consumption of antioxidants was previously shown in patients with peripheral arterial disease after ischemia-reperfusion as well as after vascular surgery (Berg et al. 2005; Wonisch et al. 2005). It should be mentioned that interpretation of data on total serum antioxidant capacity is not easy, as it is not certain which antioxidants have major impact on the overall capacity under different circumstances of acute or chronic oxidative stress. Nevertheless, these assays are useful screening methods and should be combined with other test evaluating pathology of oxidative 
A

Total capacity of antioxidants (TCA)

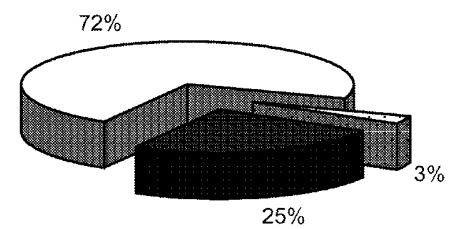

mise $\square$ Fall $\square$ No change

C

Titer of antibodies to oLDL (OLAb)

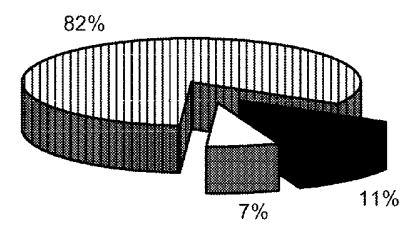

- Rise $\quad$ afall $\boldsymbol{D T}$ Transient Decrease
B

Total peroxides (POX)

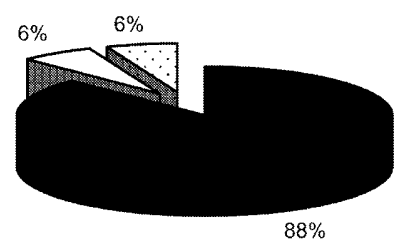

-Rise $\square$ Fall $\square$ No change

D

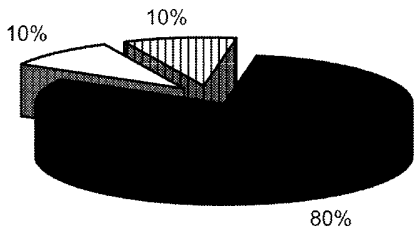

- Rise $\quad$ afall $\mathbf{0}$ Transient Decrease

Fig. 3. Patient distribution according to observed patterns of dynamic change of parameters analysed. Comparison of the data obtained in time points OV and IV were done considering also dynamic changes between these two time points. Two to three fold increase of total peroxides $(88 \%$ of patients) and sICAM-1 (80\%) was predominant pattern of dynamic change. The majority of patients $(72 \%)$ exhibited a fall in the total capacity of antioxidants. Eighty-two percent showed a transient fall in the levels of antibodies to oLDL followed by the raise of the antibody titer.

stress, as was done in the present study. In our patients, the drop in antioxidant capacity occurred at two and at four hours following PCI. However, because initial values of total antioxidant capacity were above normal range at the time of admission, the decreased values of antioxidant capacity observed after PCI still remained within the boundaries of normal values. Lysis of erythrocytes and epithelium develop following myocardial infarction (Simic et al. 2003) allowing uncontrolled leakage of intracellular antioxidants into the blood stream and thus increasing the amounts of antioxidants found in peripheral blood sera. It is also likely that aspirin as well as heparin injection could influence antioxidant capacity in time points OV, I and II, although the most recent data (Tasaki et al. 2006) suggest that heparin-released extracellular superoxide dismutase is significantly reduced in patients with coronary artery disease and that the tissue-bound location of this enzyme might be important for antioxidant function rather than its serum values. In case of heparin treatment and the tissue damage due to AMI and PCI treatment, heparin-released extracellular superoxide dismutase could be important parameter of the total serum antioxidant capacity. However, on the third and the seventh day the measured antioxidant capacity should not be affected much by heparin administered at the time surgical intervention.

The increase in the total antioxidant capacity of the sera from the cubital vein on the third day following PCI could also be due to uric acid, which is intensively produced during intensive physical stress and ischemia-reperfusion, as shown in the course of oxidative stress in marathon runners (Liu et al. 1999; Waring et al. 2003). The rise in antioxidant capacity could also be due to the release of intracellular antioxidants caused by tissue damage during AMI. Oxidative stress 
caused by AMI could afterwards reduce the serum antioxidant capacity, while PCI stabilised the condition preventing further progression of the affected tissue damage. However, it should also be mentioned that PCI itself might be a cause of oxidative stress, at least because it allows tissue reperfusion, i.e., reoxigenation. The rise of total capacity of antioxidants on the third day was probably the result of stabilised conditions associated with the food consumption that allowed an intake of antioxidants. On the other hand, a decrease of total antioxidant capacity observed afterwards, on the seventh day, could reflect physical stress due to increased physical activity of the patients, which may under convalescence resemble intense physical exercise as observed for healthy sportsmen who also exert a decrease in total antioxidant capacity after exercise (Liu et al. 1999; Schippinger et al. 2002).

A transient decrease of the oLAb titer observed in our study is consistent with the findings of Schumacher et al. (1995). A transient decrease of oLAb titer followed by an increase of the antibody titer was associated with successful convalescence after AMI (Schumacher et al. 1995), while lethal outcome was associated with continuous decrease of the anti-oLDL antibody titer. The change in titer of anti-oLDL antibodies should be primarily interpreted as a consequence of oxidative stress and subsequent lipid peroxidation. The aldehydic end products of lipid peroxidation (malondialdehyde and 4-hydroxynonenal) that are generated during oxidative stress modify proteins in the LDL molecule (apoB), generating novel epitopes for antibodies against oLDL (Jürgens et al. 1987; Esterbauer et al. 1992). Therefore, the presence of a high oLAb titer indicates an immune response against the radical attack, while a decrease of anti-oLDL antibodies after AMI reflects their protective role as scavengers for oLDL released from the culprit lesion of AMI. Moreover, as oLDL contains oxidatively modified epitopes on apoB that work as self antigens, oLDL is also an immune booster for oLDL that causes an increased titer of antibodies against oLDL several days after AMI, as observed also for our patients.

Oxidized LDL in the wall of blood vessels is removed by phagocytes. Thus activated macrophages are further source of ROS enhancing the persistence of oxidative stress, while products of lipid peroxidation act as proinflammatory factors, signalling molecules and second toxic messengers of free radicals (Vrkic et al. 1997; Forman and Torres 2001; Zarkovic 2003). Therefore, observed rise in the titer of oLAb might also reflect an inflammatory component of convalescence. This possibility is supported by data obtained for sICAM-1 results at days three and seven. The rise of sICAM-1 has already been found to be associated with AMI (Siminiak et al. 1997) also as a consequence of injury during myocardial ischemia and reperfusion (Frangogiannis et al. 2002). The raise of sICAM-1 levels was described in patients with unstable angina pectoris, but not in patients with stable angina (Kaikita et al. 1997; Ogawa et al. 1999). Therefore, it is not surprising that sICAM-1 values were increased in our patients. The increase in the levels of sICAM-1 observed in our study confirms the inflammatory pathophysiology of myocardial ischemiareperfusion injury not only during AMI and PCI but also convalescence. It is probable that this rise of sICAM-1 reflected an inflammatory vascular process after AMI and PCI, i.e., during convalescence. This possibility seems credible because an almost identical pattern of progression of levels of sICAM-1 and total peroxides $(r=0.92)$ has been observed in our patients indicating a strong link between the two parameters. The determination of serum peroxides might therefore be simple and useful method for the estimation of the persistence of systemic oxidative stress during convalescence following severe acute disorders in cardiovascular systems, as also suggested by Lindschinger et al. (2004).

Finally, it should be mentioned that several animal and clinical studies suggest that antioxidant administration might be an attractive approach to attenuate extensive oxidative attack within vital organs (Sisto et al. 1995; Metin et al. 2002; Liu et al. 2004; Kanter et al. 2005). 
Medicaments that suppress inflammatory response to the damage, as recently described for trimetazidine (Kuralay et al. 2006), could be of additional interest, while the use of aspirin known as anti-inflammatory drug that has anticoagulating and antioxidant features should be considered with care (Hobikoglu et al. 2005). Therefore, novel bioengineered compounds should be tested as well as cocktails of classical antioxidants in order to reduce the reperfusion injury within the myocardium (Hung et al. 2001), which may become efficient supportive treatments for myocardial recovery after AMI and successful convalescence of patients.

\section{Acknowledgments}

This study was supported by the Croatian Ministry of Science, Education and Sports and by the COST Action B35. The authors express their sincere gratitude to Prof. H. Sinzinger for helpful discussions and to Mrs. Tea Vukovic and Mrs. Nevenka Hirsl for excellent technical assistance. We would also like to express our gratitude to the reviewers for very constructive suggestions during the revision of the manuscript.

\section{References}

Akar, F.G., Aon, M.A., Tomaselli, G.F. \& O'Rourke, B. (2005) The mitochondrial origin of postischemic arrhythmias. J. Clin. Inv., 115, 3527-3585.

Berg, K., Jynge, P., Bjerve, K., Skarra, S., Basu, S. \& Wiseth, R. (2005) Oxidative stress and inflammatory response during and following coronary interventions for acute myocardial infarction. Free Rad. Res., 39, 629-636.

Bir, L. S., Demir, S., Rota, S. \& Koseoglu, M. (2006) Increased serum malondialdehyde levels in chronic stage of ischemic stroke. Tohoku J. Exp. Med., 208, 33-39.

Crequeira, M.D., Weissman, N.J. \& Dilsizian, V. (2002) Standardized myocardial segmentation and nomenclature for tomographic imaging of the heart: a statement for healthcare professionals from the Cardiac Imaging Committee of the Council on Clinical Cardiology of the American Heart Association. Circulation, 105, 539-542.

Esterbauer, E., Waeg, G., Puhl, H., Dieber-Rothender, M. \& Tatzber, F. (1992) Inhibition of LDL oxidation by antioxidants. EXS, 62, 145-157.

Ferrari, R., Ceconi, C., Curello, S., Alfieri, O. \& Visoli, O. (1993) Myocardial damage during ischaemia and reperfusion. Eur. Heart. J., 14G, 25-30.

Forman, H.J. \& Torres, M. (2001) Redox signalling in macrophages. Mol. Aspect. Med., 22, 189-216.

Frangogiannis, N.G., Smith, C.W. \& Entman, N.L. (2002) The inflammatory response in myocardial infarction. Cardiovasc. Res., 53, 31-47.

Frei, B. (1994) Reactive oxygen species and antioxidant vitamins. Mechanisms of action. Am. J. Med., 97, 5-12.
Hobikoglu, G.F., Norgaz, T., Aksu, H., Ozer, O., Erturk, M., Nurkalem, Z. \& Narin, A. (2005) High frequency of aspirin resistance in patients with acute coronary syndrome. Tohoku J. Exp. Med., 207, 59-64.

Hung, L.M., Chen, J.K., Lee, R.S., Liang, H.C. \& Su, M.J. (2001) Beneficial effects of astrinigin, a resverartrol analogue, on the ischemia and reperfusion damage in rat heart. Free Rad. Biol. Med., 30, 877-883.

Jürgens, G., Hoof, H., Chisolm, G.M., Cole, T.B. \& Esterbauer, H. (1987) Modification of human serum low-density lipoprotein by oxidation characterization and pathophysiological implications. Chem. Phys. Lipids., 45, 315-336.

Kaikita, K., Ogawa, H., Yasue, H., Sakamoto, T., Miyao, Y., Suefuji, H., Soejima, H., Tayama, S., Hayasaki K., Honda, T. \& Kamijikkoku, S. (1997) Increased plasma soluble intercellular adhesion molecule-1 levels in patients with acute myocardial infarction. Jap. Circul. J., 61, 741-748.

Kanter, M., Coskun, O., Amrutcu, F., Uz, Y.H. \& Kizilay, G. (2005) Protective effects of vitamin C, alone or in combination with vitamin $\mathrm{A}$, on endotoxin-induced oxidative renal tissue damage in rats. Tohoku J. Exp. Med., 206, 155-162.

Kuralay, F., Altekin, E., Yazlar, A.S., Onvural, B. \& Goldeli, O. (2006) Suppression of angioplasty-related inflammation on by pre-procedural treatment with trimetazidine. Tohoku J. Exp. Med., 208, 203-212.

Lindschinger, M., Madlinger, K., Adelwöhrer, N., Holweg, K., Wögerbauer, M., Birkmayer, J., Smolle, K.H. \& Wonisch, W. (2004) Oxidative stress: potential of distinct peroxide determination systems. Clin. Chem. Lab. Med., 42, 907-914.

Liu, M.L., Bergholm, R., Makimattila, S., Lahdenpera, S., Valkonen, M., Hilden, H., Yki-Jarvinen, H. \& Taskinen, M.R. (1999) A marathon run increases the susceptibility of LDL oxidation in vitro and modifies plasma antioxidants. Am. J. Physiol., 276, 1083-1091.

Liu, P., Xu, B., Cavalieri, T.A. \& Hock, C.E. (2004) Attenuation of antioxidative capacity enhances reperfusion injury in age rat myocardium after MI/R. Am. J. Physiol. Heart Circ. Physiol., 287, H2719-H2727.

Madamanchi, N.R., Vendrov, A., \& Runge, M.S. (2005) Oxidative stress and vascular disease. Arterioscler. Thromb. Vasc. Biol., 25, 29-38.

Metin, G., Atukeren, P., Gumustas, M.K., Belce, A. \& Kayserilioglu, A. (2002) The effect of vitamin E treatment on oxidative stress generated in trained rats. Tohoku J. Exp. Med., 198, 47-53.

Ogawa, H., Yasue, H., Miyao, Y., Sakamoto, T., Soejima, H., Nishiyama, K., Kaikita, K., Suefuji, H., Misumi, K., Takazoe, K., Kiyotaka, K. \& Yoshimura, M. (1999) Plasma soluble intercellular adhesion molecule-1 levels in coronary circulation in patients with unstable angina. Am. J. Card., 83, 38-42.

Ozbay, B. \& Dulker, H. (2002) Lipid peroxidation and antioxidant enzymes in Turkish population: relation to age, gender, exercise, and smoking. Tohoku J. Exp. Med., 197, 119-124.

Resch, U., Tatzber, F., Budinsky, A. \& Sinzinger, H. (2006) Reduction of oxidative stress and modulation of autoantibodies against modified low-density lipoprotein after rosuvastatin therapy. Br. J. Clin. Pharmacol., 61, 262-274.

Simic, D., Mimic-Oka, J., Pljesa, M., Milanovic, D., Radojevic, S., Ivanovic, B., Kalimanovska-Ostric, D., Matic, D. \& Simic, T. (2003) Time course of erythrocyte antioxidant 
activity in patients treated by thrombolysis for acute myocardial infarction. Jpn. Heart J., 44, 823-832.

Schumacher, M., Eber, B., Tatzber, F., Kaufmann, P., Halwachs, G., Frühwald, F.M., Zweiker, R., Esterbauer, H. \& Klein, W. (1995) Transient reduction of autoantibodies against oxidized LDL in patients with acute myocardial infarction. Free Rad. Biol. Med., 18, 1087-1091.

Schippinger, G., Wonisch, W., Abuja, P.M., Fankhauser, F., Winklhofer-Roob, B.M. \& Halwachs, G. (2002) Lipid peroxidation and antioxidant status in professional American football players during competition. Eur. J. Clin. Invest., 32, 686-692.

Siminiak, T., Dye, J.F., Egdell, R.M., More, R., Wysocki, H. \& Sheridan, D.J. (1997) The release of soluble adhesion molecule and E-selectin after acute myocardial infarction and following coronary angioplasty. Cardiology, 61, 113-118.

Sisto, T., Paajanen, H., Metsa-Ketela, T., Harmoinen, A., Nordback, I. \& Tarkka, M. (1995) Pretreatment with antioxidants and allopurinol diminishes cardiac onset events in coronary artery bypass grafting. Ann. Thorac. Surg., 59, 1519-1523.

Steinbrecher, U.P. (1987) Oxidation of human low-density lipoprotein results in derivatisation of lysine residues in apolipoprotein B by lipid peroxide decomposition products. J. Biol. Chem., 262, 3603-3608.

Tasaki, H., Yamashita, K., Tsutsui, M., Kamezaki, F., Kubara, T., Tanaka, S., Sasaguri, Y., Adachi, T. \& Nakashima, Y. (2006) Heparin-released extracellular superoxide dismutase is reduced in patients with coronary artery atherosclerosis. Atherosclerosis, 187, 131-138.

Tatzber, F. \& Esterbauer, H. (1995) Autoantibodies to oxidized low-density lipoprotein, In: Free Radicals IX, edited by G. Bellomo, G. Finardi, E. Maggi \& C. Rice-Evans., Richelieu Press, London, pp. 245-262.
Tatzber, F., Griebenow, S., Wonisch, W. \& Winkler, R. (2003) Dual method for the determination of peroxidase activity and total peroxides - iodide leads to a significant increase of peroxidase activity in human sera. Anal. Biochem., 316, $147-153$.

Vaarala, O. (2000) Antibodies to oxidized LDL. Lupus, 9, 202-205.

Van der Meer, I, de Maat, M.P., Bots, M.L. Breteler, M.B., Meijer, J., Kiliaan, A.J., Hofman, A. \& Witteman, J.C.M. (2002) Inflammatory mediators and cell adhesion molecules as indicators of severity of atherosclerosis: The Rotterdam Study. Arterioscler Thromb. Vasc. Biol., 22, 838-842.

Vrkic, N., Zarkovic, N., Nikolic-Heitzler, V., Topic, E., Tatzber, F., Vukelic, N. \& Kalisnik, T. (1997) Monitoring of oxidative stress markers in patients with acute myocardial infarction. Acta Clin. Croat., 36, 85-88.

Waring, W.S., Convery, A., Mishra, V., Shenkin, A., Webb, D.J. \& Maxwell, S.R. (2003) Uric acid reduces exercise induced oxidative stress in healthy adults. Clin. Sci. (Lond), 105, 425-430.

Wonisch, W., Uhl, K., Schimetta, W. \& Rabl, H. (2005) Preoperative delivery of a vitamin cocktail diminished oxidative stress after vascular surgery in PAD patients-a pilot investigation. Biofactors, 24, 299-303.

Zarkovic, N. (2003) 4-Hydroxynonenal as a bioactive marker of pathopysiological processes. Mol. Asp. Med., 24, 281-291.

Zarkovic, N., Mahovic, D., Poljak-Blazi, M., Loncaric, I., Tatzber, F., Zarkovic, K., Petravic, D., Wonisch, W. \& Zurak, N. (2004) Changes of plasma peroxides in patients with stroke: Indices of differential systemic response to oxidative stress in patients with ischemic stroke or with intracerebral hemorrhage. Neurol. Croat., 53, 79-86. 